\title{
Visualizing Overtwisted Discs in Open Books
}

\author{
by
}

Tetsuya Ito and Keiko KaWAmuro

\begin{abstract}
We give an alternative proof of a theorem of Honda-Kazez-Matić that every non-rightveering open book supports an overtwisted contact structure. We also study two types of examples that show how overtwisted discs are embedded relative to right-veering open books.
\end{abstract}

2010 Mathematics Subject Classification: Primary 57M25, 57M27; Secondary 57M50.

Keywords: open book foliation, overtwisted disc.

\section{$\S 1$. Introduction}

In [15], we have introduced open book foliations and their basic machinery by using that of braid foliations [2-9] and showed applications of open book foliations including a self-linking number formula for general closed braids. In [16] we study the geometric structure of a 3-manifold by using open book foliations. In this note we study other applications of open book foliations. We will assume the readers are familiar with the definition and basic machinery of open book foliations as presented in [15].

One of the features of open book foliations is that one can visualize how surfaces are embedded with respect to general open books. In this paper we use this feature to illustrate overtwisted discs and give constructive methods to detect overtwisted contact structures.

We first give an alternative proof of a tightness criterion theorem by Honda, Kazez and Matić [14]: If an open book is not right-veering then it supports an overtwisted contact structure.

Communicated by K. Ono. Received June 19, 2013.

T. Ito: Research Institute for Mathematical Sciences, Kyoto University,

Kyoto, 606-8502, Japan;

e-mail: tetitoh@kurims.kyoto-u.ac.jp

K. Kawamuro: Department of Mathematics, The University of Iowa,

14 MacLean Hall, Iowa City, IA 52242-1419, USA;

e-mail: keiko-kawamuro@uiowa.edu

(c) 2014 Research Institute for Mathematical Sciences, Kyoto University. All rights reserved. 
The converse does not hold: In fact, Honda, Kazez and Matić [14] show that if a contact structure $\xi$ is supported by a non-right-veering open book $(S, \phi)$, by applying positive stabilizations to $(S, \phi)$ one can find a right-veering open book $(\widehat{S}, \widehat{\phi})$ that supports $\xi$. We concretely visualize an overtwisted disc relative to the right-veering $(\widehat{S}, \widehat{\phi})$.

Lastly, we give an infinite family of open books that are right-veering and non-destabilizable but compatible with overtwisted contact structures. This negatively answers a question of Honda, Kazez and Matić [14]. Our family generalizes the previously known examples by Lekili [19] and Lisca [20], but our proof of overtwistedness is more direct.

\section{$\S 2$. Overtwisted discs in non-right-veering open books}

Recall that an overtwisted disc is an embedded disc in a contact 3-manifold whose boundary is a limit cycle in the characteristic foliation of the disc. In particular, every overtwisted disc has Legendrian boundary. In the framework of open book foliations a transverse overtwisted disc plays a corresponding role:

Definition 2.1 ([15, Def. 4.1]). Let $D \subset M_{(S, \phi)}$ be an oriented disc whose boundary is positively braided (i.e., a transverse knot) with respect to the open book $(S, \phi)$. If the following are satisfied $D$ is called a transverse overtwisted disc:

1. $G_{--}$is a connected tree with no fake vertices.

2. $G_{++}$is homeomorphic to $S^{1}$.

3. $\mathcal{F}_{\mathrm{ob}}(D)$ contains no c-circles.

(Notations and terms like $G_{--}, G_{++}$, fake vertices and c-circles are defined in $\S 2.1$ of $[15]$.

In [15] we show that the manifold $M_{(S, \phi)}$ contains a transverse overtwisted disc if and only if the contact manifold $\left(M_{(S, \phi)}, \xi_{(S, \phi)}\right)$ contains an overtwisted disc. Hence from now on, we may not distinguish a transverse overtwisted disc and a usual overtwisted disc, and often call a transverse overtwisted disc simply an overtwisted disc.

Next we review the notion of right-veering mapping classes and then reprove Honda-Kazez-Matić's tightness criterion in Theorem 2.4.

Definition 2.2 ([14]). Let $\gamma, \gamma^{\prime}$ be oriented properly embedded arcs in the surface $S$ that start from the same point $* \in \partial S$. Suppose that, after some isotopy relative to the endpoints, $\gamma$ and $\gamma^{\prime}$ realize the minimal geometric intersection number. We say that $\gamma^{\prime}$ lies strictly on the right side of $\gamma$ if it does so around the common starting point $*$. In that case we write $\gamma>\gamma^{\prime}$. 
Definition 2.3 ([14, Definition 2.1]). Let $C$ be a boundary component of $S$. We say that $\phi \in \operatorname{Aut}(\mathrm{S}, \partial \mathrm{S})$ is right-veering with respect to $C$ if $\gamma \geq \phi(\gamma)$ for any isotopy classes $\gamma$ of properly embedded curves which start at a point on $C$. We say that the diffeomorphism $\phi$ (or the open book $(S, \phi)$ ) is right-veering if $\phi$ is right-veering with respect to all the boundary components of $S$. In particular, the identity id $\in \operatorname{Aut}(S, \partial S)$ is right-veering.

The following theorem gives a characterization of open books supporting tight contact structures.

Theorem 2.4 ([14, Theorem 1.1]). If $\phi$ is not right-veering then $(S, \phi)$ supports an overtwisted contact structure.

Remark 2.5. Conversely, Honda, Kazez and Matić [14, p. 444] also prove that given an overtwisted contact structure $\xi$ there exists a non-right-veering open book $(S, \phi)$ supporting $\xi$; here Eliashberg's classification of overtwisted contact structures [10] plays an important role. By Eliashberg's classification, an overtwisted contact structure admits an open book which is a negative stabilization of some open book. Clearly such an open book has a non-right-veering monodromy. Therefore, a contact structure $\xi$ is tight if and only if every open book supporting $\xi$ is right-veering.

Proof of Theorem 2.4.. If $\phi$ is not right-veering, then there exists a properly embedded oriented arc $\alpha \subset S$ such that $\phi(\alpha)>\alpha$. By [14, Lemma 5.2], there exists a sequence of properly embedded oriented $\operatorname{arcs} \alpha_{0}, \ldots, \alpha_{k}$ such that

(i) $\alpha_{0}, \ldots, \alpha_{k}$ have the same initial point $n \in \partial S$,

(ii) $\phi(\alpha)=\alpha_{0}>\cdots>\alpha_{k}=\alpha$,

(iii) consecutive $\alpha_{i}$ and $\alpha_{i+1}$ have disjoint interiors and distinct terminal points $p_{i}, p_{i+1}$

Since $\phi\left(\alpha_{k}\right)=\alpha_{0}$ and $\phi=$ id near the binding we have $p_{0}=p_{k}$. We may assume that:

(iv) the terminal points $p_{0}, p_{1}, \ldots, p_{k-1} \in \partial S$ are mutually distinct.

Let $\beta_{i}$ (resp. $\check{\beta}_{i}$ ) be a subarc of $\alpha_{i}$ whose endpoints are $p_{i}$ and a point very close to $p_{i}$ (resp. $n$ ). See Figure 1. We orient $\alpha_{i}$ against the parametrization, i.e., the positive direction of $\alpha_{i}$ is from $p_{i}$ to $n$. The orientation of $\alpha_{i}$ induces those of $\beta_{i}$ and $\check{\beta}_{i}$. We define sets of oriented $\operatorname{arcs}$ for $i=0, \ldots, k$ :

$$
A_{i}=\beta_{0} \cup \cdots \cup \beta_{i-1} \cup \alpha_{i} \cup \beta_{i+1} \cup \cdots \cup \beta_{k} .
$$




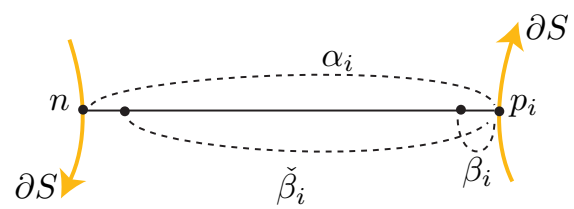

Figure 1. Arcs $\alpha_{i}, \beta_{i}$, and $\breve{\beta}_{i}$ oriented from $p_{i}$ to $n$.

Let $t_{i}=i / k \in[0,1]$. In the following, we construct an oriented surface $D_{i}$ properly embedded in the product region $S \times\left[t_{i}, t_{i+1}\right]$, where $i=0, \ldots, k-1$, such that $D_{i} \cap S_{t_{i}}=-A_{i}$ and $D_{i} \cap S_{t_{i+1}}=A_{i+1}$.

The surface $D_{i}$ consists of $k$ connected components; one non-product region and $k-1$ product regions defined by $\beta_{j} \times\left[t_{i}, t_{i+1}\right]$ where $j \neq i, i+1$ and $0 \leq j \leq k$.

In the open book foliation of $D_{i}$ the point $n$ becomes a negative elliptic point, the points $p_{0}, \ldots, p_{k-1}$ become positive elliptic points, and the $\operatorname{arc} \beta_{j} \times\{t\}$ becomes an a-arc in the page $S_{t}$. (See [15, Prop. 2.2] for the definition of a-arcs.) The nonproduct component of $D_{i}$ is defined by the movie presentation as sketched in Figure 2. It is a saddle shape surface with a positive hyperbolic point $h_{i}$.
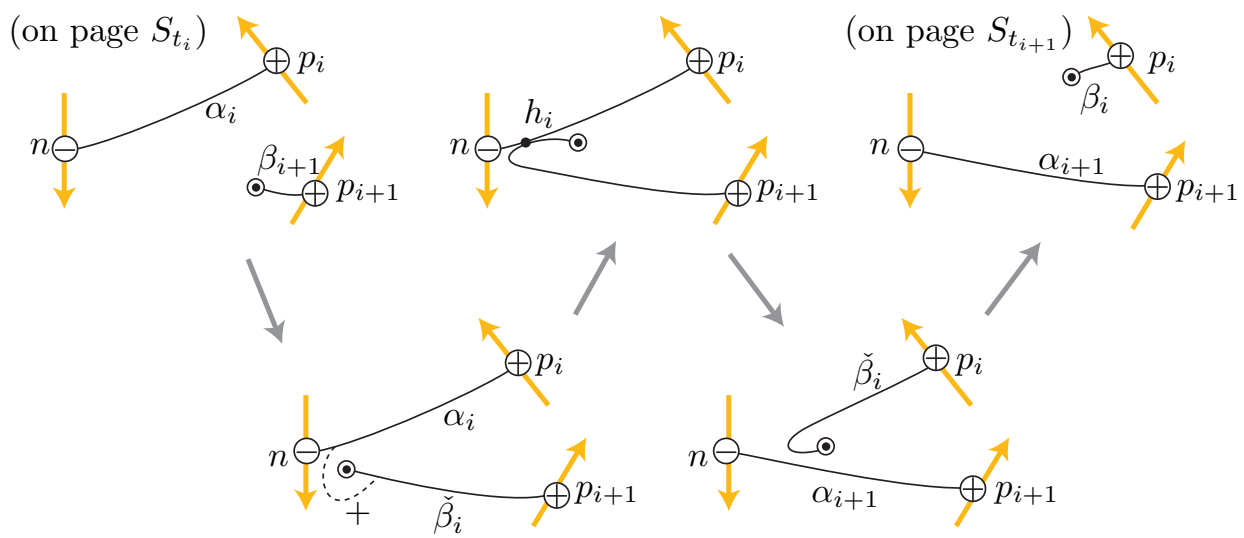

Figure 2. The non-product region of surface $D_{i}$ (movie presentation).

Now we glue $D_{i}$ and $D_{i+1}$ along $A_{i+1} \subset S_{t_{i+1}}(i=0, \ldots, k-2)$ and obtain a surface $D_{0} \cup \cdots \cup D_{k-1} \subset S \times[0,1]$ whose oriented boundary is $\left(-A_{0}\right) \cup A_{k}$. Since the $\operatorname{arcs} \beta_{1}, \ldots, \beta_{k}$ are very close to $\partial S$ and $\phi=$ id near $\partial S$, we have $A_{0}=\phi\left(A_{k}\right)$. So in the manifold $M_{(S, \phi)}$ we can identify $A_{0}$ and $A_{k}$ and obtain a surface which we denote by $D$. 


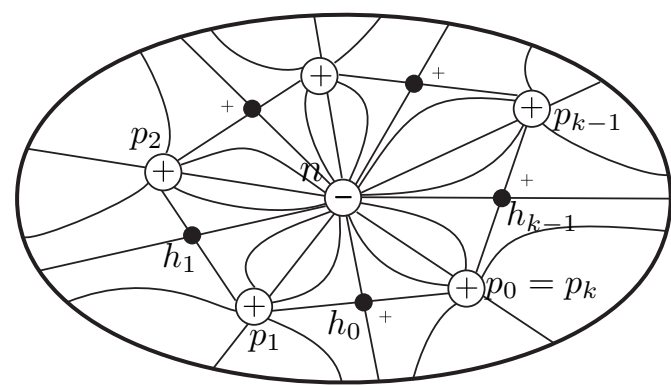

Figure 3. The transverse overtwisted disc $D$.

The topological type of $D$ is the disc and its open book foliation $\mathcal{F}_{\mathrm{ob}}(D)$ is depicted in Figure 3. Clearly our $D$ is a transverse overtwisted disc.

Remark 2.6. Honda, Kazez and Matić's proof and our proof are based on the same combinatorial lemma [14, Lemma 5.2] in order to use the assumption of right-veeringness. Our proof is more elementary and different from the original one that is written in the language of convex surface theory:

In [14], the authors prove the existence of a bypass, half of an overtwisted disc, by applying [14, Lemma 5.2] and the right-to-life principle [12, Lemma 2.9], [13, Proposition 2.2] which involves the Legendrian realization principle and Eliashberg's classification of tight contact structures on the 3-ball.

On the other hand, we use [14, Lemma 5.2] to explicitly construct a chain of positive elliptic and hyperbolic points surrounding the central negative elliptic point of an overtwisted disc. Hence our proof concretely visualizes an overtwisted disc.

\section{$\S 3$. Overtwisted discs in right-veering open books}

The converse of Theorem 2.4 does not hold in general. Honda, Kazez and Matić [14] show that every contact structure is supported by a right-veering open book.

Their argument is the following: Given a contact structure $(M, \xi)$ choose a compatible open book $(S, \phi)$. For a boundary component $C$ of $S$ take two boundary-parallel arcs $a_{C}$ and $b_{C}$ such that the geometric intersection number $i\left(a_{C}, b_{C}\right)$ is 2 . Apply positive stabilizations to $(S, \phi)$ along $a_{C}$ and $b_{C}$ for all the boundary components $C$ on which $\phi$ is non-right-veering. The new open book $(\widehat{S}, \widehat{\phi})$ is now right-veering (see [14, Prop. 6.1]), and supports the same contact structure $\xi$. 
Suppose that we start from a non-right-veering open book $(S, \phi)$, hence $\xi$ is overtwisted. In the following we concretely describe how an overtwisted disc is embedded with respect to the stabilized right-veering open book $(\widehat{S}, \widehat{\phi})$.

Example 3.1 (Overtwisted discs in Honda-Kazez-Matić's stabilizations). Let $(S, \phi)$ be a non-right-veering open book. By the proof of Theorem 2.4 one can construct an overtwisted disc, $D$, in $M_{(S, \phi)}$. The open book foliation of $D$ has a unique negative elliptic point, say $n$, that lies on the binding component $C \subset \partial S$, and $k$ positive elliptic points $p_{1}, \ldots, p_{k}$ and $k$ positive hyperbolic points $h_{1}, \ldots, h_{k}$. Let $S_{t_{i}}$ be the singular fiber that contains $h_{i}$. We may assume

$$
0<t_{1}<\cdots<t_{k}<1 / 2<1 \text {. }
$$

For $t \in[0,1)$ let $b_{t} \in S_{t} \cap D$ be the b-arc (cf. [15, Prop. 2.2]) that ends at the point $n$.

Now we apply Honda-Kazez-Matić's stabilization to get a right-veering open book $(\widehat{S}, \widehat{\phi})$. The monodromy $\widehat{\phi}$ satisfies $\widehat{\phi}=T_{\beta} \circ T_{\alpha} \circ \phi$, where $\alpha$ and $\beta$ are core circles of the annuli plumbed along $a_{C}$ and $b_{C}$, and $T_{\alpha}, T_{\beta}$ are positive Dehn twists along $\alpha, \beta$.

We will construct an overtwisted disc $\widehat{D}$ by giving a movie presentation relative to the open book $(\widehat{S}, \widehat{\phi})$. For simplicity we assume that:

- $\phi$ is non-right-veering only along $C$.

- $p_{k} \in \partial S \backslash C$.

In the general case the construction of $\widehat{D}$ is similar but more complicated. It is obtained as an application of arguments in [17].

Choose stabilization $\operatorname{arcs} a_{C}$ and $b_{C}$ such that $i\left(\alpha, b_{1 / 2}\right)=1$ and $i\left(\beta, b_{1 / 2}\right)=0$ as shown in Figure 4(a). Such arcs can always be found by the assumptions above.

To the region $\left\{\widehat{S}_{t} \mid t \in[0,1 / 2]\right\}$ add a continuous family of b-arcs that are co-cores of the annuli plumbed along $a_{C}$ (see Figure 4(a)). We denote the positive and negative elliptic points which are the endpoints of the newly added b-arcs by $p^{\prime}$ and $n^{\prime}$. Except for this family of b-arcs, the movie presentation of $\widehat{D}$ in the interval $[0,1 / 2]$ is the same as that of $D$. Let $\widehat{b}_{t}:=b_{t}$.

In the interval $[1 / 2,1), \widehat{D}$ is described as in the passage $(\mathrm{b}) \rightarrow(\mathrm{c}) \rightarrow(\mathrm{d})$ of Figure 4 . We form one negative and one positive hyperbolic point, $h_{-}$and $h_{+}$, as in (b) and (c), respectively. The describing arcs of $h_{-}$and $h_{+}$are parallel to $\alpha$. We have $\widehat{b}_{1}=T_{\alpha}^{-1}\left(b_{1}\right)$. Note that $\widehat{\phi}\left(\widehat{b}_{1}\right)=\left(T_{\beta} \circ T_{\alpha} \circ \phi\right)\left(\widehat{b}_{1}\right)=T_{\beta} \circ T_{\alpha}\left(T_{\alpha}^{-1} b_{0}\right)=$ $T_{\beta}\left(b_{0}\right)=b_{0}=\widehat{b}_{0}$, moreover $\widehat{\phi}\left(\widehat{D} \cap S_{1}\right)=\widehat{D} \cap S_{0}$ so this movie presentation indeed defines an overtwisted disc in $M_{(\widehat{S}, \widehat{\phi})}$. 

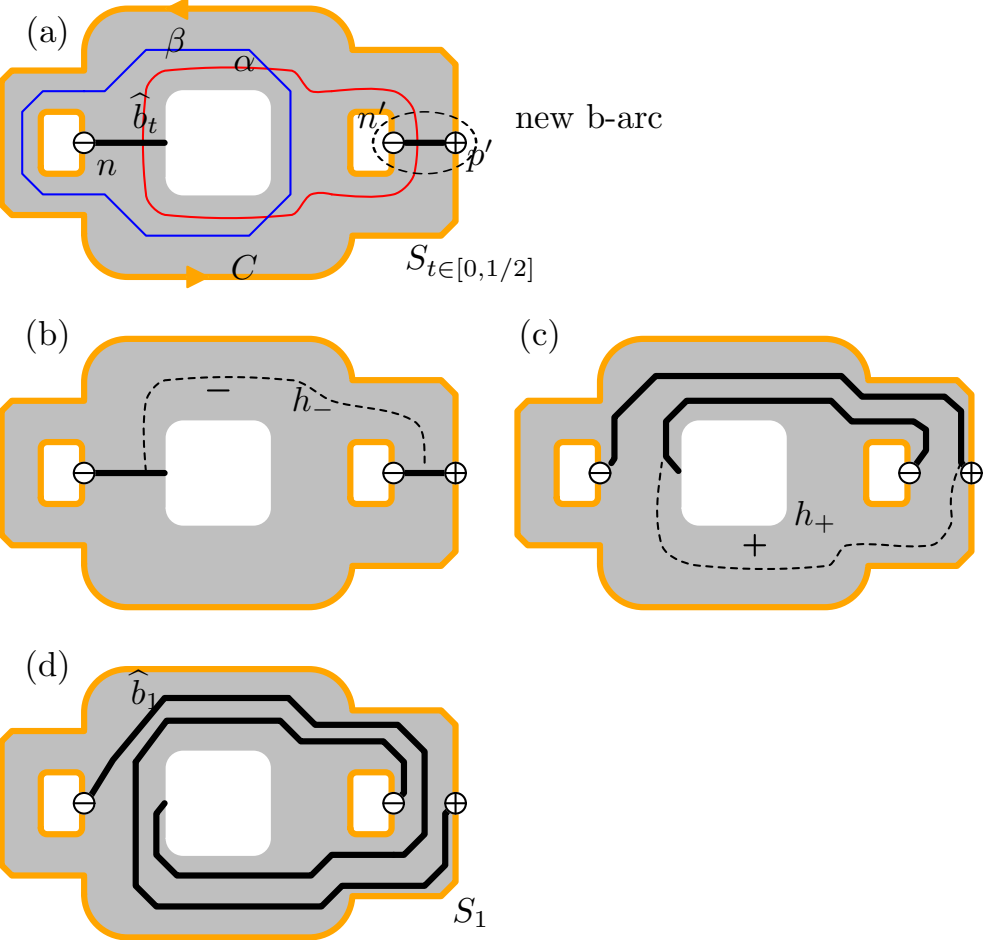

Figure 4. A movie presentation of the overtwisted disc $\widehat{D}$. The shaded region is the union of a collar neighborhood of $C$ and the two plumbed annuli. All the a-arcs are omitted for simplicity.
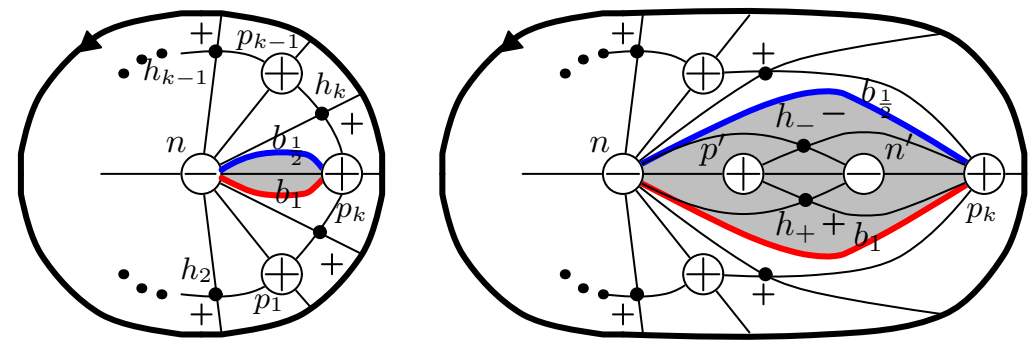

Figure 5. $\mathcal{F}_{\mathrm{ob}}(D)$ and $\mathcal{F}_{\mathrm{ob}}(\widehat{D})$.

The open book foliations of the overtwisted discs $D$ and $\widehat{D}$ are depicted in Figure 5, where $\mathcal{F}_{\mathrm{ob}}(\widehat{D})$ is obtained by inserting two bb-tiles of opposite signs into the shaded region of $\mathcal{F}_{\mathrm{ob}}(D)$ that is bounded by $b_{1 / 2}$ and $b_{1}$. 
Strictly speaking, the disc $\widehat{D}$ is not a transverse overtwisted disc since condition (3) of Definition 2.1 is not satisfied. However, applying the same technique as in the alternative proof in [15] of the Bennequin-Eliashberg inequality [1, 11], condition (3) will be satisfied. Thus we can regard $\widehat{D}$ as an overtwisted disc.

\section{$\S 4$. Generalization of Lekili's and Lisca's examples}

In [14, Question 6.2] Honda, Kazez and Matić ask whether a right-veering and non-destabilizable open book always supports a tight contact structure. Lekili [19] and Lisca [20] negatively answer the question by constructing examples. They study open book decompositions of 3-manifolds whose tight contact structures are well-studied and classified (in [19] Poincaré homology 3-spheres, and in [20] lens spaces). In both constructions the most technical points are showing that their open books indeed support overtwisted contact structures. Advanced tools such as Ozsváth-Szábo's Heegaard Floer invariants and properties of planar open books enable them to overcome the difficulty.

We generalize Lekili's and Lisca's examples in Theorem 4.1 below. Our proof of overtwistedness is direct and does not require any knowledge of classification of tight contact structures of ambient manifolds or Ozsváth-Szábo's invariants.

Theorem 4.1. Let $S$ be a 2-sphere with four holes. Let $a, b, c, d, e$ be simple closed curves on $S$ as shown in Figure 6 . Let $\Phi_{h, i, k}=T_{a}^{h} T_{b}^{i} T_{c} T_{d} T_{e}^{-k-1}$ where $T_{x}(x=$ $a, b, c, d, e)$ denotes the right-handed Dehn twist along $x$. Then for all $h, i, k \geq 1$, $\Phi_{h, i, k}$ is right-veering and the open book $\left(S, \Phi_{h, i, k}\right)$ is non-destabilizable and supports an overtwisted contact structure.

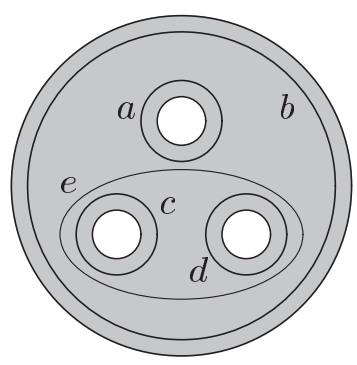

Figure 6. The surface $S$.

Remark 4.2. Lekili's examples [19, Theorem 1.2 and Remark 4.1] are $\Phi_{2, i, 1}$ $(i \leq 5)$, and Lisca's examples [20, Theorem 1.1] are $\Phi_{h, 1, l}(h, l>0)$. 
Proof of Theorem 4.1. We owe to [19], [20] the proof that $\Phi_{h, i, k}$ is right-veering and non-destabilizable. Hence we only show that $\left(S, \Phi_{h, i, k}\right)$ supports an overtwisted contact structure. We define a transverse overtwisted disc $D$ in the open book $\left(S, \Phi_{h, i, k}\right)$ by the movie presentation as in sketches (1)-(7) of Figure 7 . For example, sketch (1) depicts the page $S_{0}$ with the set of arcs $D \cap S_{0}$. On $\partial S$ there are two negative elliptic points $n_{1}, n_{2}$, and $2 k+3$ positive elliptic points $p_{1}, \ldots, p_{2 k+3}$. The movie presentation shows that $\mathcal{F}_{\mathrm{ob}}(D)$ contains two negative hyperbolic points and $2 k+3$ positive hyperbolic points. Note that $\Phi_{h, i, k}\left(D \cap S_{1}\right)=D \cap S_{0}$.

The corresponding open book foliation $\mathcal{F}_{\mathrm{ob}}(D)$ is depicted in Figure 8. We can verify that $D$ meets the conditions in Definition 2.1 of transverse overtwisted discs.

(1)

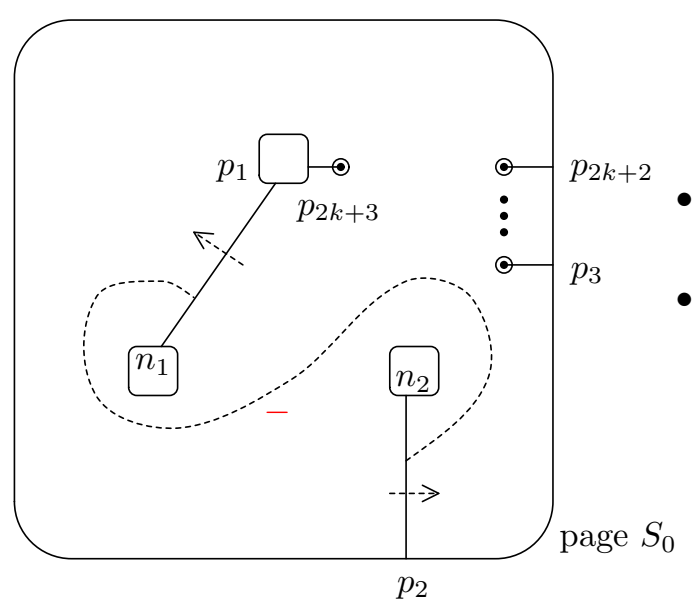

(2)

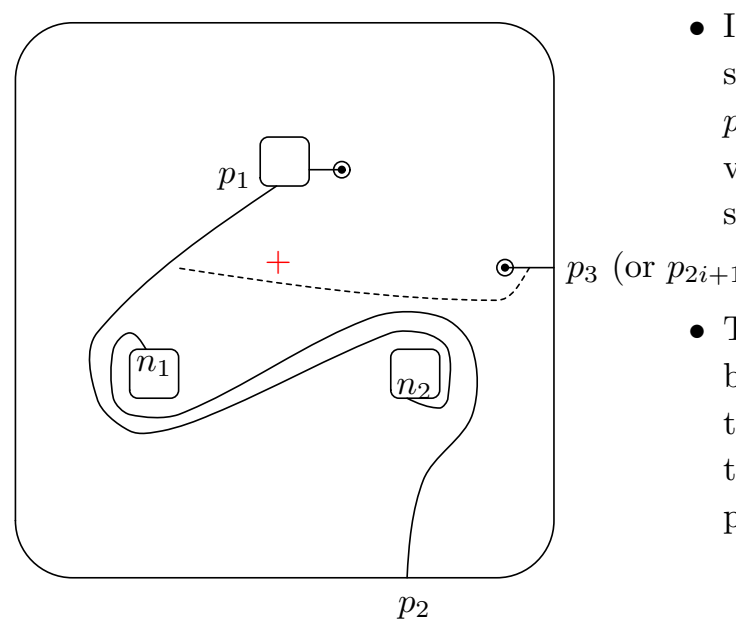

- Leaves in the fiber $S_{0}$ consist of two b-arcs and $2 k+1$ a-arcs.

- The dashed describing arc corresponds to a negative hyperbolic point.
- In sketches $(2), \ldots,(7)$, we omit some a-arcs emanating from $p_{3}, \ldots, p_{2 k+2}$ if they are not involved in producing hyperbolic singularities.

- The describing arc joining the b-arc emanating from $n_{2}$ and the a-arc from $p_{3}$ (or $p_{2 i+1}$ in the $i$-th iteration) represents a positive hyperbolic point. 
(3)

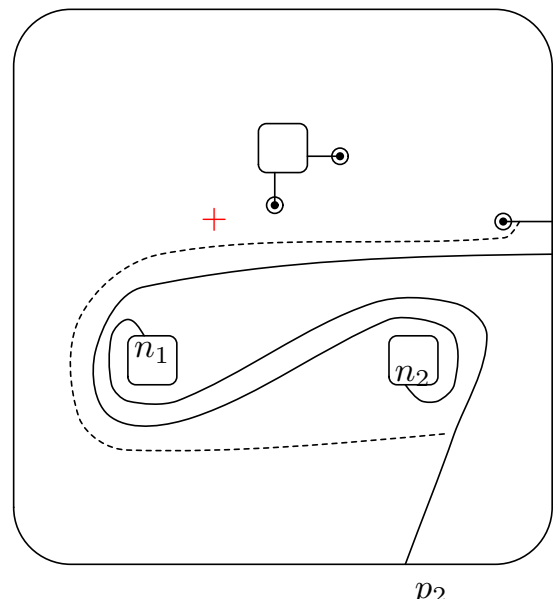

$p_{4}\left(\right.$ or $\left.p_{2 i+2}\right)$

$p_{3}\left(\right.$ or $\left.p_{2 i+1}\right)$

- The describing arc joining the b-arc emanating from $n_{1}$ and the a-arc from $p_{4}$ (or $p_{2 i+2}$ in the $i$-th iteration) represents a positive hyperbolic point.

- Iterate steps (2) and (3) $k$ times.

(4)

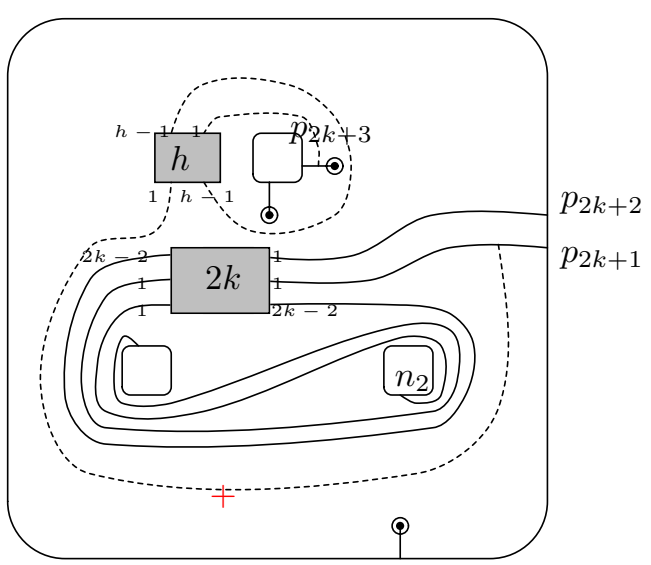

- The shaded boxes labeled $h, 2 k$ contain parallel $h$ and $2 k$ arcs.

- The edges out of the shaded boxes are weighted as indicated.

- The describing arc joins the barc from $n_{2}$ to $p_{2 k+1}$ and the aarc emanating from $p_{2 k+3}$ and it represents a positive hyperbolic point.

(5)

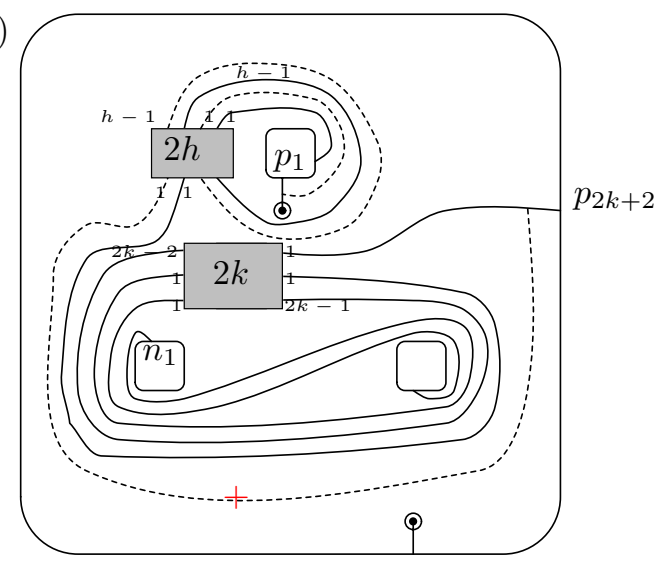

- The describing arc joins the barc from $n_{1}$ to $p_{2 k+2}$ and the a-arc emanating from $p_{1}$ and it represents a positive hyperbolic point. 
(6)

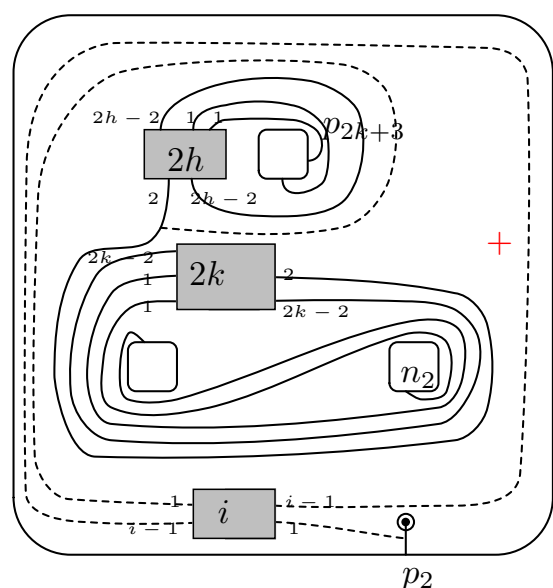

(7)

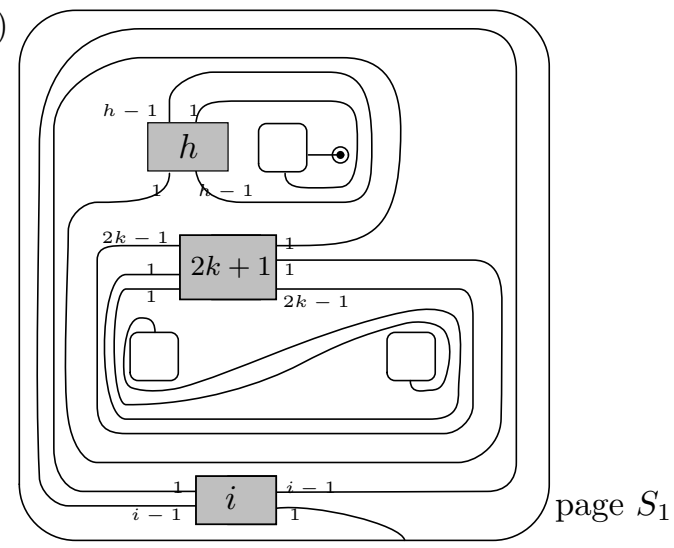

- The describing arc joins the barc from $n_{2}$ to $p_{2 k+3}$ and the a-arc emanating from $p_{2}$ and it represents a positive hyperbolic point.

- The leaves in the page $S_{1}$. It satisfies $\Phi_{h, i, k}\left(D \cap S_{1}\right)=D \cap S_{0}$.

Figure 7. Movie presentation of a transverse overtwisted disc in $\left(S, \Phi_{h, i, k}\right)$.

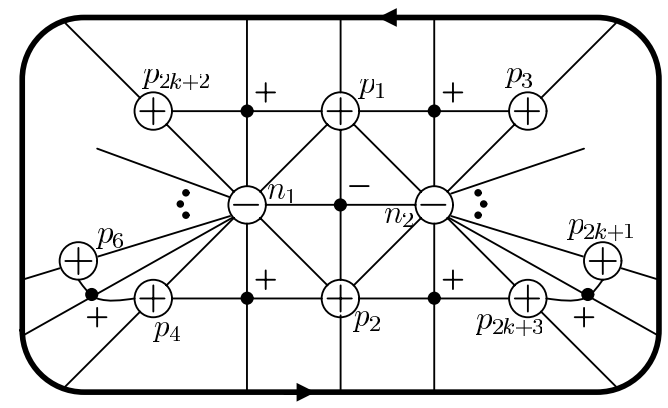

Figure 8. The open book foliation $\mathcal{F}_{\mathrm{ob}}(D)$ 
Remark 4.3. After the announcement of this example (on December 26, 2011), Kazez and Roberts [18] found more counterexamples to the conjecture of Honda, Kazez and Matić.

\section{Acknowledgements}

The first author was supported by JSPS Research Fellowships for Young Scientists. The second author was partially supported by NSF grants DMS-0806492 and DMS-1206770.

\section{References}

[1] D. Bennequin, Entrelacements et équations de Pfaff, Astérisque 107-108 (1983), 87-161. Zbl 0573.58022 MR 0753131

[2] J. Birman and W. Menasco, Studying links via closed braids. IV. Composite links and split links, Invent. Math. 102 (1990), 115-139. Zbl 0711.57006 MR 1069243

[3] - Studying links via closed braids. II. On a theorem of Bennequin, Topology Appl. 40 (1991), 71-82. Zbl 0722.57001 MR 1114092

[4] _ Studying links via closed braids. V. The unlink, Trans. Amer. Math. Soc. 329 (1992), 585-606. Zbl 0758.57005 MR 1030509(92g:57010b)

[5] _ Studying links via closed braids. I. A finiteness theorem, Pacific J. Math. 154 (1992), 17-36. Zbl 0724.57001 MR 1154731

[6] Studying links via closed braids. VI. A nonfiniteness theorem, Pacific J. Math. 156 (1992), 265-285. Zbl 0739.57002 MR 1186805

[7] _ Studying links via closed braids. III. Classifying links which are closed 3-braids, Pacific J. Math. 161 (1993), 25-113. Zbl 0813.57010 MR 1237139

[8] $\_$Stabilization in the braid groups. I. MTWS, Geom. Topol. 10 (2006), 413-540. Zbl 1128.57003 MR 2224463

[9] Stabilization in the braid groups. II. Transversal simplicity of knots, Geom. Topol. 10 (2006), 1425-1452. Zbl 1130.57005 MR 2255503

[10] Y. Eliashberg, Classification of overtwisted contact structures on 3-manifolds, Invent. Math. 98 (1989), 623-637. Zbl 0684.57012 MR 1022310

[11] Contact 3-manifolds twenty years since J. Martinet's work, Ann. Inst. Fourier (Grenoble) 42 (1992), 165-192. Z Zbl 0756.53017 MR 1162559

[12] K. Honda, On the classification of tight contact structures I, Geom. Topol. 4 (2000), 309368. Zbl 0980.57010 MR 1786111

[13] K. Honda, W. Kazez and G. Matić, Tight contact structures on fibered hyperbolic 3manifolds, J. Differential Geom. 64 (2003), 305-358. Zbl 1083.53082 MR 2029907

[14] Right-veering diffeomorphisms of compact surfaces with boundary, Invent. Math. 169 (2007), 427-449. Zbl 1167.57008 MR 2318562

[15] T. Ito and K. Kawamuro, Open book foliations, Geom. Topol., to appear.

[16] Essential open book foliation and fractional Dehn twist coefficient, arXiv:1208.1559.

[17] _ Operations on open book foliations, Algebr. Geom. Topol., to appear; arXiv:1309.4486.

[18] W. Kazez and R. Roberts, Fractional Dehn twists in knot theory and contact topology, Algebr. Geom. Topol. 13 (2013), 3603-3637. Zbl 06216310

[19] Y. Lekili, Planar open books with four binding components, Algebr. Geom. Topol. 11 (2011) 909-928. Zbl 1220.57017 MR 2782547

[20] P. Lisca, On overtwisted, right-veering open books, Pacific J. Math. 257 (2012), 219-225. Zbl 1254.57022 MR 2948466 